\title{
RAMANUJAN, HIS LOST NOTEBOOK, ITS IMPORTANCE
}

\author{
BRUCE C. BERNDT
}

\section{Brief Biography of Ramanujan}

Throughout history most famous mathematicians were educated at renowned centers of learning and were taught by inspiring teachers, if not by distinguished research mathematicians. The one exception to this rule is Srinivasa Ramanujan, born on December 22, 1887 in Erode in the southern Indian State of Tamil Nadu. He lived most of his life in Kumbakonam, located to the east of Erode and about 250 kilometers south, southwest of Madras, or Chennai as it is presently named. At an early age, he won prizes for his mathematical ability, not mathematics books as one might surmise, but books of English poetry, reflecting British colonial rule at that time. At the age of about fifteen, he borrowed a copy of G. S. Carr's Synopsis of Pure and Applied Mathematics, which was the most influential book in Ramanujan's development. Carr was a tutor and compiled this compendium of approximately 4000-5000 results (with very few proofs) to facilitate his tutoring at Cambridge and London. One or two years later, Ramanujan entered the Government College of Kumbakonam, often called "the Cambridge of South India," because of its high academic standards. By this time, Ramanujan was consumed by mathematics and would not seriously study any other subject. Consequently he failed his examinations at the end of the first year and lost his scholarship. Because his family was poor, Ramanujan was forced to terminate his formal education.

At about the time Ramanujan entered college, he began to record his mathematical discoveries in notebooks. Living in poverty with no means of financial support, suffering at times from serious illnesses (including two long bouts of dysentery), and working in isolation, Ramanujan devoted all of his efforts in the next five years to mathematics, while continuing to record his theorems without proofs in notebooks.

In 1909, Ramanujan married S. Janaki, who was only nine years old. More pressure was therefore put upon him to find a job, and so in 1910 he arranged a meeting with V. Ramaswami Aiyar, who had founded the Indian Mathematical Society three years earlier and who was working as a Deputy Collector in the Civil Service. After Ramanujan showed Ramaswami Aiyar his notebooks, the latter contacted R. Ramachandra Rao, Collector in the town of Nellore, north of Madras, who agreed to provide Ramanujan with a monthly stipend so that he could continue to work unceasingly on mathematics and not worry about employment.

In 1910, with the financial support of Ramachandra Rao, Ramanujan moved to Madras. For reasons that are unclear, after fifteen months, Ramanujan declined further support and subsequently became a clerk in the Madras Port Trust Office, where 
he was encouraged, especially by Sir Francis Spring and S. Narayana Aiyar, Chairman and Chief Accountant, respectively. Narayana Aiyar held a Master's Degree in Mathematics and at that time was probably one of the most capable mathematicians in India [20]. They persuaded Ramanujan to write English mathematicians about his mathematical discoveries. One of them, G. H. Hardy, responded encouragingly and invited Ramanujan to come to Cambridge to develop his mathematical gifts. Ramanujan's family were Iyengars, a conservative orthodox branch in the Brahmin tradition, and especially his mother was adamantly opposed to her son's "crossing the seas" and thereby becoming "unclean." After overcoming family reluctance, Ramanujan boarded a passenger ship for England on March 17, 1914.

At about this time, Ramanujan evidently stopped recording his theorems in notebooks. That Ramanujan no longer concentrated on logging entries in his notebooks is evident from two letters that he wrote to friends in Madras during his first year in England [31, pp. 112-113; 123-125]. In a letter of November 13, 1914 to his friend R. Krishna Rao, Ramanujan confided, "I have changed my plan of publishing my results. I am not going to publish any of the old results in my notebooks till the war is over." And in a letter of January 7, 1915 to S. M. Subramanian, Ramanujan admitted, "I am doing my work very slowly. My notebook is sleeping in a corner for these four or five months. I am publishing only my present researches as I have not yet proved the results in my notebooks rigorously."

On March 24, 1915, near the end of his first winter in Cambridge, Ramanujan wrote to his friend E. Vinayaka Row [31, pp. 116-117] in Madras, "I was not well till the beginning of this term owing to the weather and consequently I couldn't publish any thing for about 5 months." By the end of his third year in England, Ramanujan was critically ill, and, for the next two years, he was confined to nursing homes. After World War I ended, Ramanujan returned home in March, 1919, but his health continued to deteriorate, and on April 26, 1920 Ramanujan died at the age of 32.

In both England and India, Ramanujan was treated for tuberculosis, but his symptoms did not match those of the disease. More recently, an English physician, D. A. B. Young [32, pp. 65-75] carefully examined all extant records and symptoms of Ramanujan's illness and convincingly concluded that Ramanujan suffered from hepatic amoebiasis, a parasitic infection of the liver. Amoebiasis is a protozoal infection of the large intestine that gives rise to dysentery. Relapses occur when the host-parasite relationship is disturbed, which likely happened when Ramanujan entered a colder climate. The illness is very difficult to diagnose, but once diagnosed, it can be cured.

Despite being confined to nursing homes for two of his five years in England, Ramanujan made enormously important contributions to mathematics, several in collaboration with Hardy, which, although they won him immediate and lasting fame, are probably recognized and appreciated more so today than they were at that time. Most of Ramanujan's discoveries lie in the areas of (primarily) number theory, analysis, and combinatorics, but they influence many modern branches of both mathematics and physics. 


\section{History of Ramanujan's Notebooks}

After Ramanujan died, Hardy strongly urged that Ramanujan's notebooks be edited and published. Hardy used the word "edit" in the broader sense to indicate that each claim made by Ramanujan in his notebooks should be examined and proved, if a proof did not already exist. Of the three notebooks that Ramanujan left us, the second is the most extensive and is actually a revision of the first, although there are over 100 entries in the first notebook that cannot be found in the second. The third notebook has only 33 pages. Altogether, the notebooks contain about 3200-3300 different entries, almost all of them without proofs and most of them original discoveries. We do not know for certain when Ramanujan began to record his results in notebooks, but as mentioned above, we think his compilation began in about 1904 when he entered college; as the letters above indicate, he ceased to record his theorems in notebooks when he departed for England in 1914.

At the University of Madras, various papers and handwritten copies of all three notebooks were sent to Hardy in 1923 with the intent of bringing together all of Ramanujan's work for publication. It transpired that Ramanujan's Collected Papers [61] were published in 1927, but his notebooks and other manuscripts were not published.

Sometime in the late 1920s, two English mathematicians, G. N. Watson and B. M. Wilson, undertook the task of editing Ramanujan's notebooks. Wilson died prematurely in 1935, and although Watson worked for 10-15 years on the task and wrote over 30 papers inspired by Ramanujan's mathematics, the work was never completed.

It was not until 1957 that the notebooks were made available to the public when the Tata Institute of Fundamental Research in Bombay published a photocopy edition [62], but no editing was undertaken.

In February, 1974 while reading two papers by Emil Grosswald [47], [48], in which some formulas from the notebooks were proved, the present author observed that he could prove these formulas by using a theorem he had proved two years earlier and so was naturally curious to determine if there were other formulas in the notebooks that could be proved using his theorem. He was at the Institute for Advanced Study in Princeton at that time, and fortunately, the library at Princeton University had a copy of the Tata Institute's edition. He found a few more formulas, similar to those that he had already proven, but he found a few thousand other formulas that he could not prove. In the following two years the author wrote two long papers [12], [13] on series identities and evaluations in the spirit of those formulas from the notebooks that Grosswald had first proved.

All of the aforementioned entries can be found in Chapter 14 of Ramanujan's second notebook. After the spring semester at the University of Illinois ended in May, 1977, the author decided to attempt to find proofs for all 87 formulas in Chapter 14. He worked on this project for over a year, and then G. E. Andrews from Pennsylvania State University visited the University of Illinois and informed him that on a visit to Trinity College Library at Cambridge two years earlier, he learned that Watson and Wilson's efforts in editing the notebooks were preserved there. The librarian at Trinity College kindly sent the author a copy of these notes, and so with their help on certain chapters, he began to devote all of his research time toward proving the theorems stated 
by Ramanujan in his three notebooks. With the assistance of several mathematicians, the task was completed in five volumes [14]-[18] over a period of 21 years.

Why did not Ramanujan record any of his proofs in his three notebooks? There are perhaps several reasons. First, Ramanujan was perhaps influenced by the style of Carr's book in which one theorem after another is stated without proofs. Second, like most Indian students in his time, Ramanujan worked primarily on a slate. Paper was expensive, and so writing out all of his proofs on paper would have been prohibitive for Ramanujan. Therefore, after rubbing out the proofs on his slate with his sleeve, Ramanujan recorded only the final results in his notebooks. Third, Ramanujan probably felt that there was no need to record his arguments. If someone asked him how to prove a particular result in his notebooks, he undoubtedly could supply his proof. He had never intended that his notebooks would be made available to the mathematical public. They were his own personal compilation of what he had discovered. It has also been proposed that Ramanujan did not provide proofs because he was following the long-standing Indian tradition of stating mathematical facts without arguments or proofs. However, there is no evidence that Ramanujan had studied ancient Indian mathematics. With India being under British colonial rule at that time, Ramanujan was more influenced by English mathematics than by traditional Indian mathematics. In fact, Ramanujan's early papers and problems that he contributed to the Journal of the Indian Mathematical Society were in the same milieu as the mathematics being studied and created by other Indian mathematicians in the period around 1910 . We emphasize, however, that Ramanujan's work did not remain at this level, as he quickly took the modest amount of mathematics that he knew and developed it in many directions, so that mathematicians today continue to build along the paths that he started.

Speculations about Ramanujan's methods and proofs are plentiful. Many have suggested that he discovered his results by "intuition," or by making deductions from numerical calculations, or by inspiration from Goddess Namagiri. Indeed, like many mathematicians, Ramanujan evidently made extensive calculations. However, explanations emphasizing "intuition," religion, or Eastern mysticism are of no value. Ramanujan's idea of a proof arose from the British tradition and not from the venerable Indian tradition. Because of his lack of a rigorous training in mathematics, as Ramanujan himself was aware, some of his arguments were not rigorous by then contemporary standards. Nonetheless, despite his lack of rigor at times, Ramanujan doubtless thought and devised proofs like any other mathematician, but with insights that surpass all but a few of the greatest mathematicians.

\section{Discovery of the Lost Notebook}

As mentioned above, in the spring of 1976, Andrews visited Trinity College Library at Cambridge University. Dr. Lucy Slater had suggested to him that there were materials deposited there from the estate of the late G. N. Watson that might be of interest to him. In one box of materials from Watson's estate, Andrews found several items written by Ramanujan, with the most interesting item being a manuscript written on 138 sides in Ramanujan's distinctive handwriting. The sheets contained over six 
hundred formulas without proofs. Although technically not a notebook, and although technically not "lost," as we shall see in the sequel, it was natural in view of the fame of Ramanujan's (earlier) notebooks [62] to call this manuscript Ramanujan's lost notebook. Almost certainly, this manuscript, or at least most of it, was written during the last year of Ramanujan's life, after his return to India from England.

The manuscript contains no introduction or covering letter. In fact, there are hardly any words in the manuscript. There are a few marks evidently made by a cataloguer, and there are also a few remarks in the handwriting of Hardy. Undoubtedly, the most famous objects examined in the lost notebook are the mock theta functions, about which more will be written later.

The natural, burning question now is: How did this manuscript of Ramanujan come into Watson's possession? We think that the manuscript's history can be traced.

\section{History of the Lost Notebook}

After Ramanujan died on April 26, 1920, his notebooks and unpublished papers were given by his widow, Janaki, to the University of Madras. (It should be remarked that in a conversation with the author, Janaki told him that during the funeral of her late husband, many of his papers were stolen by two certain people. These stolen papers have never been located.) Also at that time, Hardy strongly advocated bringing together all of Ramanujan's manuscripts, both published and unpublished, for publication. On August 30, 1923, Francis Dewsbury, the registrar at the University of Madras, wrote to Hardy informing him that [31, p. 266]:

I have the honour to advise despatch to-day to your address per registered and insured parcel post of the four manuscript note-books referred to in my letter No. 6796 of the 2nd idem.

I also forward a packet of miscellaneous papers which have not been copied. It is left to you to decide whether any or all of them should find a place in the proposed memorial volume. Kindly preserve them for ultimate return to this office.

(Hardy evidently never returned any of the miscellaneous papers.) Although no accurate record of this material exists, the amount sent to Hardy was doubtless substantial. It is therefore highly likely that this "packet of miscellaneous papers" contained the aforementioned "lost notebook." R. A. Rankin, in fact, opines [32, p. 124]:

It is clear that the long MS represents work of Ramanujan subsequent to January 1920 and there can therefore be little doubt that it constitutes the whole or part of the miscellaneous papers dispatched to Hardy from Madras on 30 August 1923.

Further details can be found in Rankin's accounts of Ramanujan's unpublished manuscripts [65], [66], [32, pp. 117-142].

In 1934, Hardy passed on to Watson a considerable amount of his material on Ramanujan. However, it appears that either Watson did not possess the "lost" notebook in 1936 and 1937 when he published his papers [70], [71] on mock theta functions, or he had not thoroughly examined it. In any event, Watson [70, p. 61], [31, p. 330] writes 
that he believes that Ramanujan was unaware of certain third order mock theta functions and transformation formulas for the fifth order mock theta functions. But, in his lost notebook, Ramanujan did indeed examine these third order mock theta functions, and found transformation formulas for the fifth order mock theta functions. Watson's interest in Ramanujan's mathematics waned in the late 1930s, and Hardy died in 1947. In conclusion, sometime between 1934 and 1947 and probably closer to 1947, Hardy gave Watson the manuscript that we now call the "lost notebook."

Watson was Mason Professor of Pure Mathematics at the University of Birmingham for most of his career, retiring in 1951. He died in 1965 at the age of 79. Rankin, who succeeded Watson as Mason Professor but who had since become Professor of Mathematics at the University of Glasgow, was asked to write an obituary of Watson for the London Mathematical Society. Rankin wrote [65], [32, p. 120]:

For this purpose I visited Mrs Watson on 12 July 1965 and was shown into a fair-sized room devoid of furniture and almost knee-deep in manuscripts covering the floor area. In the space of one day I had time only to make a somewhat cursory examination, but discovered a number of interesting items. Apart from Watson's projected and incomplete revision of Whittaker and Watson's Modern Analysis in five or more volumes, and his monograph on Three decades of midland railway locomotives, there was a great deal of material relating to Ramanujan, including copies of Notebooks 1 and 2, his work with B. M. Wilson on the Notebooks and much other material. ... In November 191965 Dr J. M. Whittaker who had been asked by the Royal Society to prepare an obituary notice [72], paid a similar visit and unearthed a second batch of Ramanujan material. A further batch was given to me in April 1969 by Mrs Watson and her son George.

Since her late husband had been a Fellow and Scholar at Trinity College and had had an abiding, lifelong affection for Trinity College, Mrs. Watson agreed with Rankin's suggestion that the library at Trinity College would be the most appropriate place to preserve her husband's papers. Since Ramanujan had also been a Fellow at Trinity College, Rankin's suggestion was even more appropriate.

During the next three years, Rankin sorted through Watson's papers, and dispatched Watson's and Ramanujan's papers to Trinity College in three batches on November 2, 1965; December 26, 1968; and December 30, 1969, with the Ramanujan papers being in the second shipment. Rankin did not realize the importance of Ramanujan's papers, and so when he wrote Watson's obituary [64] for the Journal of the London Mathematical Society, he did not mention any of Ramanujan's manuscripts. Thus, for almost eight years, Ramanujan's "lost notebook" and some fragments of papers by Ramanujan lay in the library at Trinity College, known only to a few of the library's cataloguers, Rankin, Mrs. Watson, Whittaker, and perhaps a few others. The 138-page manuscript waited there until Andrews found it and brought it before the mathematical public in the spring of 1976. It was not until the centenary of Ramanujan's birth on December 22, 1987, that Narosa Publishing House in New Delhi published in photocopy form Ramanujan's lost notebook and his other unpublished papers [63]. 


\section{The Origin of the Lost Notebook}

Having detailed the probable history of Ramanujan's lost notebook, we return now to our earlier claim that the lost notebook was written in the last year of Ramanujan's life. On February 17, 1919, Ramanujan returned to India after almost five years in England, the last two being confined to nursing homes. Despite the weakening effects of his debilitating illness, Ramanujan continued to work on mathematics. Of this intense mathematical activity, up to the discovery of the lost notebook, the mathematical community knew only of the mock theta functions. These functions were described in Ramanujan's last letter to Hardy, dated January 12, 1920 [61, pp. xxix-xxx, 354-355], [31, pp. 220-223], where he wrote:

I am extremely sorry for not writing you a single letter up to now .... I discovered very interesting functions recently which I call "Mock" $\vartheta$ functions. Unlike the "False" $\vartheta$-functions (studied partially by Prof. Rogers in his interesting paper) they enter into mathematics as beautifully as the ordinary theta functions. I am sending you with this letter some examples.

In this letter, Ramanujan defines four third order mock theta functions, ten fifth order functions, and three seventh order functions. He also includes three identities satisfied by the third order functions and five identities satisfied by his first five fifth order functions. He states that the other five fifth order functions also satisfy similar identities. In addition to the definitions and formulas stated by Ramanujan in his last letter to Hardy, the lost notebook contains further discoveries of Ramanujan about mock theta functions. In particular, it contains the five identities for the second family of fifth order functions that were only mentioned but not stated in the letter.

We think that we have made the case for our assertion that the lost notebook was composed during the last year of Ramanujan's life, when, by his own words, he discovered the mock theta functions. In fact, only a fraction (perhaps 5-8\%) of the notebook is devoted to the mock theta functions themselves.

\section{General Content of the Lost Notebook}

The next fundamental question is: What is in Ramanujan's lost notebook besides mock theta functions? A majority of the results fall under the purview of $q$-series. These include mock theta functions, theta functions, partial theta functions, false theta functions, identities connected with the Rogers-Fine identity, several results in the theory of partitions, Eisenstein series, modular equations, the Rogers-Ramanujan continued fraction, other $q$-continued fractions, asymptotic expansions of $q$-series and $q$-continued fractions, integrals of theta functions, integrals of $q$-products, and incomplete elliptic integrals. Other continued fractions, other integrals, infinite series identities, Dirichlet series, approximations, arithmetic functions, numerical calculations, Diophantine equations, and elementary mathematics are some of the further topics examined by Ramanujan in his lost notebook.

The Narosa edition [63] contains further unpublished manuscripts, portions of both published and unpublished papers, letters to Hardy written from nursing homes, and 
scattered sheets and fragments. The three most famous of these unpublished manuscripts are those on the partition function and Ramanujan's tau function [30], [6], forty identities for the Rogers-Ramanujan functions [26], [6], and the unpublished remainder of Ramanujan's published paper on highly composite numbers [58], [61, pp. 78-128], [6].

In the passages that follow, we select certain topics and examples to illustrate the content and importance of Ramanujan's discoveries found in his lost notebook. Andrews and the author are in the process of writing five volumes on the lost notebook that are analogous to those that the author wrote on the earlier notebooks [14]-[18]. At the moment of this writing, four of the volumes [4]-[7] have been published.

\section{7. $q$-SERIES AND Theta Functions}

The vast majority of entries in Ramanujan's lost notebook are on $q$-series. Thus, we should begin the more technical portion of this essay by giving a brief introduction to $q$-series. Generally, a $q$-series has expressions of the type

$$
(a)_{n}:=(a ; q)_{n}:=(1-a)(1-a q) \cdots\left(1-a q^{n-1}\right), \quad n \geq 0,
$$

in the summands, where we interpret $(a ; q)_{0}=1$. If the base $q$ is understood, we often use the notation at the far left-hand side of (7.1). Series with factors $(a ; q)_{n}$ in their summands are also called Eulerian series. Our definition of a $q$-series is not entirely satisfactory, because often in the theory of $q$-series, we let parameters in the summands tend to 0 or to $\infty$, and consequently it may happen that no factors of the type $(a ; q)_{n}$ remain in the summands. In some cases, what may remain is a theta function. Following the lead of Ramanujan, we define a general theta function $f(a, b)$ by

$$
f(a, b):=\sum_{n=-\infty}^{\infty} a^{n(n+1) / 2} b^{n(n-1) / 2}, \quad|a b|<1 .
$$

Perhaps the most useful property of theta functions is the famous Jacobi triple product identity [16, p. 35, Entry 19] given by

$$
f(a, b)=(-a ; a b)_{\infty}(-b ; a b)_{\infty}(a b ; a b)_{\infty}, \quad|a b|<1,
$$

where

$$
(a ; q)_{\infty}:=\lim _{n \rightarrow \infty}(a ; q)_{n}, \quad|q|<1 .
$$

For this exposition, in Ramanujan's notation and with the use of (7.3), only one special case,

$$
f(-q):=f\left(-q,-q^{2}\right)=\sum_{n=-\infty}^{\infty}(-1)^{n} q^{n(3 n-1) / 2}=(q ; q)_{\infty},
$$

is relevant for us. If $q=e^{2 \pi i \tau}$, where $\operatorname{Im} \tau>0$, then $q^{1 / 24} f(-q)=\eta(\tau)$, the Dedekind eta function. The last equality in (7.4) renders Euler's pentagonal number theorem. For a more detailed introduction to $q$-series, see the author's paper [19]. 


\section{Mock Theta Functions}

As indicated above, mock theta functions are certain kinds of $q$-series first introduced by Ramanujan in his last letter to Hardy written on 12 January 1920 . He begins his letter by examining the asymptotic behavior of two $q$-series as $t \rightarrow 0$, where $q=e^{-t}$. For example,

$$
\sum_{n=0}^{\infty} \frac{q^{n^{2}}}{(q ; q)_{n}^{2}}=\sqrt{\frac{t}{2 \pi}} \exp \left(\frac{\pi^{2}}{6 t}-\frac{t}{24}\right)+o(1),
$$

as $t \rightarrow 0$. The series above is the reciprocal of a theta function, namely, it is the reciprocal of $f(-q)$, defined in (7.4). Ramanujan then asks if the converse is true. That is, suppose we have a $q$-series that exhibits an asymptotic behavior of the kind described in (8.1) as we approach any exponential singularity $e^{2 \pi i m / n}$ of the function. Must the function actually be a theta function plus some easily described trivial function? Ramanujan says "not necessarily so." "When it is not so I call the function Mock $\vartheta$-function."

Ramanujan then gives several examples of mock theta functions. For example, he defines

$$
f(q):=\sum_{n=0}^{\infty} \frac{q^{n^{2}}}{(-q ; q)_{n}^{2}}
$$

and asserts that

$$
f(q)+\sqrt{\frac{\pi}{t}} \exp \left(\frac{\pi^{2}}{24 t}-\frac{t}{24}\right) \rightarrow 4
$$

as $t \rightarrow 0$, with $q=e^{-t}$. Then he remarks, "It is inconceivable that a single $\vartheta$ function could be found to cut out the singularities of $f(q)$. (The definition of $f(q)$ in $(8.2)$ has no relation with the function $f(-q)$ defined in (7.4).) Thus, $f(q)$ is a mock theta function (of the third order). What is the order of a mock theta function? Ramanujan does not tell us. We emphasize that Ramanujan does not prove that $f(q)$ is actually a mock theta function according to his somewhat imprecise definition. Moreover, no one since has actually proved this statement, nor has anyone proved that any of Ramanujan's mock theta functions are really mock theta functions according to his definition. Note that the series on the left side of (8.1) is similar in appearance to the series defining $f(q)$ in (8.2); only the signs of the parameters in the summands' $q$-products are different. However, the two series behave quite differently, both analytically and arithmetically in regard to their coefficients. Indeed, one of the fascinating features of $q$-series is that making what appears to be a small modification in the series terms drastically alters the behavior of the function. Only recently, A. Folsom, K. Ono, and R. C. Rhoades [45] established for the first time that the limit on the right-hand side of (8.3) is indeed equal to 4.

Ramanujan's lost notebook contains many identities involving mock theta functions. We offer two identities for fifth order mock theta functions:

$$
\chi(q):=\sum_{n=0}^{\infty} \frac{q^{n}}{\left(q^{n+1}\right)_{n}}=1+\sum_{n=0}^{\infty} \frac{q^{2 n+1}}{\left(q^{n+1}\right)_{n+1}}
$$


and

where

$$
f_{0}(q)+2 \Phi\left(q^{2}\right)=\frac{\left(q^{5} ; q^{5}\right)_{\infty}\left(q^{5} ; q^{10}\right)_{\infty}}{\left(q ; q^{5}\right)_{\infty}\left(q^{4} ; q^{5}\right)_{\infty}}
$$

$$
f_{0}(q):=\sum_{n=0}^{\infty} \frac{q^{n^{2}}}{(-q ; q)_{n}}, \quad \Phi(q):=-1+\sum_{n=0}^{\infty} \frac{q^{5 n^{2}}}{\left(q ; q^{5}\right)_{n+1}\left(q^{4} ; q^{5}\right)_{n}}
$$

All three functions $\chi(q), f_{0}(q)$, and $\Phi(q)$ are mock theta functions. Both of these identities have interesting implications in the theory of partitions, which we address in Section 9 .

The discovery of the lost notebook by Andrews and then the publication of the lost notebook by Narosa in 1988 [63] stimulated an enormous amount of research on mock theta functions, as researchers found proofs of the many mock theta function identities found in the lost notebook. We mention only a few of the more important contributions by: Andrews [2], [3], Andrews and F. Garvan [9], and D. Hickerson [52] on fifth order mock theta functions; Andrews and Hickerson [10] on sixth order mock theta functions; Andrews [2] and Hickerson [53] on seventh order mock theta functions; and Y.-S. Choi [37]-[40] on tenth order mock theta functions.

As we have seen, Ramanujan's definition of a mock theta function is somewhat vague. Can a precise, coherent theory be developed and find its place among the other great theories of our day? In 1987, at a meeting held at the University of Illinois commemorating Ramanujan on the centenary of his birth, F. J. Dyson addressed this question [42, p. 20], [32, p. 269].

The mock theta-functions give us tantalizing hints of a grand synthesis still to be discovered. Somehow it should be possible to build them into a coherent group-theoretical structure, analogous to the structure of modular forms which Hecke built around the old theta-functions of Jacobi. This remains a challenge for the future. My dream is that I will live to see the day when our young physicists, struggling to bring the predictions of superstring theory into correspondence with the facts of nature, will be led to enlarge their analytic machinery to include not only theta-functions but mock theta-functions. Perhaps we may one day see a preprint written by a physicist with the title "Mock Atkin-Lehner Symmetry." But before this can happen, the purely mathematical exploration of the mock-modular forms and their mock-symmetries must be carried a great deal farther.

Since we invoke the words, modular form, at times in the sequel, we provide here a brief definition. Let $V(\tau)=(a \tau+b) /(c \tau+d)$, where $a, b, c$, and $d$ are integers such that $a d-b c=1$, and where $\operatorname{Im} \tau>0$. Then $f(\tau)$ is a modular form of weight $k$ if

$$
f(V(\tau))=\epsilon(a, b, c, d)(c \tau+d)^{k} f(\tau),
$$

where $k$ is real (usually an integer or half of an integer) and $|\epsilon(a, b, c, d)|=1$.

In recent years, the work of S. Zwegers [73] and K. Bringmann and K. Ono [35], [36] has made progress in the direction envisioned by Dyson. First it was observed, to take one example, that the infinite product on the right-hand side of (8.5) essentially 
coincided with the Fourier expansion of a certain weakly holomorphic modular form, where the term "weakly holomorphic" indicates that the modular form is analytic in the upper half-plane, but may have poles at what are called "cusps" on the real axis. In his doctoral dissertation [73], Zwegers related mock theta functions to real analytic vector-valued modular forms by adding to Ramanujan's mock theta functions certain non-holomorphic functions, which are called period integrals. Earlier work of Andrews [2], who used Bailey pairs to express Ramanujan's Eulerian series in terms of Hecketype series, was also essential for Zwegers, since he applied his ideas to the Hecke-type series rather than Ramanujan's original series. Zwegers' real analytic modular forms are examples of harmonic Maass forms. Briefly, a Maass form satisfies the functional equation (8.6) with $k=0$ and is an eigenfunction of the hyperbolic Laplacian

$$
\Delta:=-y^{2}\left(\frac{\partial^{2}}{\partial x^{2}}+\frac{\partial^{2}}{\partial y^{2}}\right)
$$

where $\tau=x+i y$. A harmonic Maass form $M(\tau)$ again satisfies a functional equation of the type (8.6) when $k$ is an integer and a slightly different functional equation if $k$ is half of an integer, but the operator (8.7) is replaced by

$$
\Delta_{k}:=-y^{2}\left(\frac{\partial^{2}}{\partial x^{2}}+\frac{\partial^{2}}{\partial y^{2}}\right)+i k y\left(\frac{\partial}{\partial x}+i \frac{\partial}{\partial y}\right) .
$$

It is now required that $\Delta_{k} M=0$.

Returning to (8.2), Bringmann and Ono [36] examined the more general function

$$
R(\omega, q):=\sum_{n=0}^{\infty} \frac{q^{n^{2}}}{(\omega q ; q)_{n}\left(\omega^{-1} q ; q\right)_{n}}
$$

and proved (under certain hypotheses), when $\omega$ is a root of unity, that $R(\omega, q)$ is the holomorphic part of a weight $\frac{1}{2}$ harmonic Maass form. In general, Bringmann and Ono showed that each of Ramanujan's mock theta functions is the holomorphic part of a harmonic Maass form.

Recall that in their famous paper [50], Hardy and Ramanujan developed an asymptotic series for the partition function $p(n)$, defined to be the number of ways the positive integer $n$ can be expressed as a sum of positive integers. For example, since $4=3+1=2+2=2+1+1=1+1+1+1, p(4)=5$. Some years later, improving on their work, H. Rademacher [57] found a convergent series representation for $p(n)$. If we write the mock theta function $f(q)$ from (8.2) as $f(q)=\sum_{n=0}^{\infty} \alpha(n) q^{n}$, Andrews [1] analogously found an asymptotic series for $\alpha(n)$. Bringmann and Ono [35] were able to replace the asymptotic formula by an exact formula confirming a conjecture of Andrews.

We have sketched only a few highlights among the extensive recent developments in the theory of mock theta functions. Readers are encouraged to read Ono's comprehensive description [56] of these developments. In Ono's paper, readers will also find discussions and references to the permeation of mock theta functions in physics, thus providing evidence for Dyson's prophetic vision. 


\section{Partitions}

Recall from above the definition of the partition function $p(n)$. Inspecting a table of $p(n), 1 \leq n \leq 200$, calculated by P. A. MacMahon, Ramanujan was led to conjecture the congruences

$$
\begin{cases}p(5 n+4) & \equiv 0(\bmod 5) \\ p(7 n+5) & \equiv 0(\bmod 7) \\ p(11 n+6) & \equiv 0(\bmod 11)\end{cases}
$$

which he eventually proved [60], [63], [30]. In 1944, Dyson [41] sought to combinatorially explain (9.1) and in doing so defined the rank of a partition to be the largest part minus the number of parts. For example, the rank of $3+1$ is 1 . Dyson observed that the congruence classes for the rank modulo 5 and 7 divided the partitions of $p(5 n+4)$ and $p(7 n+5)$, respectively, into equinumerous classes. These conjectures were subsequently proved by A. O. L. Atkin and H. P. F. Swinnerton-Dyer [11]. However, for the third congruence in (9.1), the corresponding criterion failed, and so Dyson conjectured the existence of a statistic, which he called the crank to combinatorially explain the congruence $p(11 n+6) \equiv 0(\bmod 11)$. The crank of a partition was found by Andrews and Garvan [8] and is defined to be the largest part if the partition contains no one's, and otherwise to be the number of parts larger than the number of one's minus the number of one's. The crank divides the partitions into equinumerous congruence classes modulo 5, 7, and 11 for the three congruences, respectively, in (9.1).

At roughly the same time that Andrews and Garvan found the crank, it was observed that in his lost notebook, Ramanujan had found the generating functions for both the rank and the crank. First, if $N(m, n)$ denotes the number of partitions of $n$ with rank $m$, then

$$
\sum_{n=0}^{\infty} \sum_{m=-\infty}^{\infty} N(m, n) z^{m} q^{n}=\sum_{n=0}^{\infty} \frac{q^{n^{2}}}{(z q ; q)_{n}\left(z^{-1} q ; q\right)_{n}} .
$$

The definition (9.2) should be compared with that in (8.8). Second, if $M(m, n)$ denotes the number of partitions of $n$ with crank $m$, then, except for a few small values of $m$ and $n$,

$$
\sum_{n=0}^{\infty} \sum_{m=-\infty}^{\infty} M(m, n) z^{m} q^{n}=\frac{(q ; q)_{\infty}}{(z q ; q)_{\infty}\left(z^{-1} q ; q\right)_{\infty}} .
$$

We do not know if Ramanujan knew the combinatorial implications of the rank and crank. However, in view of the several results on the generating functions for the rank and crank as well as calculations for cranks found in his lost notebook, it is clear that he had realized the importance of these two functions [46], [23]. There is also evidence that his very last mathematical thoughts were on cranks before he died on April 26, 1920 [24].

Many identities in the lost notebook have partition theoretic implications. As promised earlier, we now examine the partition-theoretic interpretations of (8.4) and (8.5).

We state (8.4) in an equivalent form: The number of partitions of a positive integer $N$ where the smallest part does not repeat and the largest part is at most twice the 
smallest part equals the number of partitions of $N$ where the largest part is odd and the smallest part is larger than half the largest part. As an example, take $N=7$. Then the relevant partitions are, respectively, $7=4+3=2+2+2+1$ and $7=3+2+2=$ $1+1+1+1+1+1+1$. A short proof can be constructed with the use of Ferrers diagrams, and we leave this proof for the reader.

To examine (8.5), we first define $\rho_{0}(n)$ to be the number of partitions of $n$ with unique smallest part and all other parts $\leq$ the double of the smallest part. For example, $\rho_{0}(5)=3$, with the relevant partitions being $5,3+2$, and $2+2+1$. Second, let $N(a, b, n)$ denote the number of partitions of $n$ with rank congruent to $a$ modulo $b$. Then (8.5) is equivalent to The First Mock Theta Conjecture,

$$
N(1,5,5 n)=N(0,5,5 n)+\rho_{0}(n) .
$$

For example, if $n=5$, then $N(1,5,25)=393, N(0,5,25)=390$, and, as observed above, $\rho_{0}(5)=3$. Although (8.5) has been proved by Hickerson, and now also by A. Folsom [43] and by Hickerson and E. Mortenson [54], a combinatorial proof of (9.4) has never been given.

\section{FURTHER $q$-SERIES}

We have discussed only two facets among Ramanujan's voluminous contributions to $q$-series in his lost notebook. In this short section, we briefly provide two more examples in illustration of this abundance. One of the showpieces in the theory of $q$-series is Heine's transformation [5, p. 6, Thm. 1.2.1]

$$
\sum_{n=0}^{\infty} \frac{(a)_{n}(b)_{n}}{(c)_{n}(q)_{n}} t^{n}=\frac{(b ; q)_{\infty}(a t ; q)_{\infty}}{(c ; q)_{\infty}(t ; q)_{\infty}} \sum_{n=0}^{\infty} \frac{(c / b)_{n}(t)_{n}}{(a t)_{n}(q)_{n}} b^{n}
$$

where $|t|,|b|<1$. There are many identities in the lost notebook, whose proofs naturally use Heine's transformation [5, Chapter 1]. One consequence of (10.1) is, for $|a q|<1$ [63, p. 38],

$$
\sum_{n=0}^{\infty} \frac{(-a q)^{n}}{\left(-a q^{2} ; q^{2}\right)_{n}}=\sum_{n=0}^{\infty} \frac{(-1)^{n} a^{n} q^{n(n+1) / 2}}{(-a q ; q)_{n}} .
$$

To see how (10.1) can be used to prove (10.2), consult [5, p. 25, Entry 1.6.4]. For partition-theoretic proofs, see [33] and [27].

Our second identity is given by [63, p. 35], [5, p. 35, Entry 1.7.9]

$$
\sum_{n=0}^{\infty} \frac{(-1)^{n} q^{n(n+1) / 2}}{(q ; q)_{n}\left(1-q^{2 n+1}\right)}=f\left(q^{3}, q^{5}\right)
$$

where $f(a, b)$ is defined by (7.2). We see partitions at work on the left-hand side of (10.3), but on the right-hand side, we observe that these partitions have cancelled each other out, except on a thin set of quadratic exponents. For a combinatorial proof of (10.3), see [27]. 


\section{The Rogers-Ramanujan Continued Fraction}

As the name suggests, the Rogers-Ramanujan continued fraction

$$
R(q):=\frac{q^{1 / 5}}{1}+\frac{q}{1}+\frac{q^{2}}{1}+\frac{q^{3}}{1}+\cdots, \quad|q|<1,
$$

was first studied by L. J. Rogers [67] in 1894 and then by Ramanujan before he departed for England, for his notebooks [62] contain many properties of this continued fraction. His study of $R(q)$ continued unabatedly in the lost notebook, with the first five chapters of [4] focusing on $R(q)$. Readers may find it strange that the first numerator of $R(q)$ in (11.1) is $q^{1 / 5}$. Because $R(q)$ lives in the homes of theta functions and modular forms, once we see how it relates to the other functions living in the same homes, we understand more fully why $q^{1 / 5}$ is a part of the continued fraction.

In his first letter to Hardy [61, p. xxvii], [31, p. 29], Ramanujan offered the elegant values of

$$
R\left(e^{-2 \pi}\right)=\sqrt{\frac{5+\sqrt{5}}{2}}-\frac{\sqrt{5}+1}{2}
$$

and $R\left(-e^{-\pi}\right)$, and in his second letter, he communicated the value of $R\left(e^{-2 \pi \sqrt{5}}\right)[61$, p. xxviii], [31, p. 37]. In his lost notebook, Ramanujan recorded several further values; e.g., on page 46 of [63],

$$
R\left(-e^{-\pi \sqrt{3}}\right)=\frac{(3+\sqrt{5})-\sqrt{6(5+\sqrt{5})}}{4} .
$$

A key to evaluating $R(q)$ in closed form is the identity (found in Ramanujan's notebooks $[16$, p. 267])

$$
\frac{1}{R(q)}-1-R(q)=\frac{f\left(-q^{1 / 5}\right)}{q^{1 / 5} f\left(-q^{5}\right)},
$$

where $f(-q)$ is defined by (7.4), and a similar identity involving $R^{5}(q)$ that can be derived from (11.2). If one can evaluate the quotient of Dedekind eta functions on the right-hand side of (11.2) for a certain value of $q$, then one can determine $R(q)$ by solving a simple quadratic equation. The evaluation of quotients of eta functions at points $e^{-\pi \sqrt{n}}$ is usually quite difficult. To this end, evaluating appropriate class invariants, which are certain quotients of eta functions at $e^{-\pi \sqrt{n}}$, is often helpful. The first general theorem for determining values of $R(q)$ in this direction was developed by the author, H. H. Chan, and L.-C. Zhang [25].

The function $R(q)$ satisfies several beautiful modular equations. For example [4, p. 92], if $u=R(q)$ and $v=R\left(q^{2}\right)$, then

$$
\frac{v-u^{2}}{v+u^{2}}=u v^{2}
$$

Because of limitations of space, we must desist from providing further properties for $R(q)$, but in closing we remark that $R(q)$ also serves as a model, in that its properties guided Ramanujan and others that followed in their quests of finding analogous theorems for other $q$-continued fractions. In particular, see Chapters 6-8 in [4]. 


\section{Eisenstein Series}

Continuing in his lost notebook the study of Eisenstein series made in [59], [61, pp. 136-162], Ramanujan offers many further discoveries about these series. Furthermore, published with his lost notebook are several letters that Ramanujan wrote to Hardy from nursing homes during his last two years in England; these letters are primarily devoted to Eisenstein series. An account of all of these discoveries can be found in the last six chapters in [5]. In this section, we briefly discuss some of these results.

In Ramanujan's notation, the three primary Eisenstein series are

$$
\begin{aligned}
& P(q):=1-24 \sum_{k=1}^{\infty} \frac{k q^{k}}{1-q^{k}}, \\
& Q(q):=1+240 \sum_{k=1}^{\infty} \frac{k^{3} q^{k}}{1-q^{k}}, \\
& R(q):=1-504 \sum_{k=1}^{\infty} \frac{k^{5} q^{k}}{1-q^{k}} .
\end{aligned}
$$

For $q=\exp (2 \pi i \tau)$, $\operatorname{Im} \tau>0$, in more contemporary notation, $Q(q)=E_{4}(\tau)$ and $R(q)=E_{6}(\tau)$. These two functions are modular forms of weights 4 and 6 , respectively, and are the "building blocks" for modular forms on the full modular group. The function $P(q)$ is not a modular form, but is a quasi-modular form, because it satisfies the same transformation formulas as an ordinary modular form.

As emphasized in Section 9, Hardy and Ramanujan [50], [61, pp. 276-309] found an asymptotic series for the partition function $p(n)$, which arises from the power series coefficients of the reciprocal of the Dedekind eta-function. As they indicated near the end of their paper, their methods also apply to coefficients of further modular forms that are analytic in the upper half-plane. In their last jointly published paper [51], [61, pp. 310-321], they considered a similar problem for the coefficients of modular forms having a simple pole in a fundamental region, and, in particular, they applied their theorem to find interesting series representations for the coefficients of the reciprocal of the Eisenstein series $E_{6}(\tau)$. In letters from nursing homes, Ramanujan calculated formulas for the coefficients of further quotients of Eisenstein series. The formulas, which were first proved in papers by the author with P. Bialek [21] and with Bialek and A. J. Yee [22], do not fall under the purview of the general theorem from [51]. As they are too complicated to offer in a short survey, we invite readers to examine them in the aforementioned papers or to consult [5, Chapter 11].

Ramanujan recorded many beautiful identities for Eisenstein series in [63]. We close this section with one of them [5, p. 331]. If $f(-q)$ is defined by $(7.4)$, then

$$
Q(q)=\frac{f^{10}(-q)}{f^{2}\left(-q^{5}\right)}+250 q f^{4}(-q) f^{4}\left(-q^{5}\right)+3125 q^{2} \frac{f^{10}\left(-q^{5}\right)}{f^{2}(-q)} .
$$

\section{The Circle and Divisor Problems}

Let $r_{2}(n)$ denote the number of representations of the positive integer $n$ as a sum of two squares. The famous circle problem is to determine the precise order of magnitude 
for the "error term" $P(x)$ defined by

$$
\sum_{0 \leq n \leq x}^{\prime} r_{2}(n)=\pi x+P(x)
$$

as $x \rightarrow \infty$, where the prime $/$ on the summation sign on the left side indicates that if $x$ is an integer, only $\frac{1}{2} r_{2}(x)$ is counted. We now explain why this problem is called the circle problem. Each representation of $n$ as a sum of two squares can be associated with a lattice point in the plane. For example, $5=(-2)^{2}+1^{2}$ can be associated with the lattice point $(-2,1)$. Then each lattice point can be associated with a unit square, say that unit square for which the lattice point is in the southwest corner. Thus, the sum in (13.1) is equal to the number of lattice points in the circle of radius $\sqrt{x}$ centered at the origin, or to the sum of the areas of the aforementioned squares, and the area of this circle, namely $\pi x$, is a reasonable approximation to the sum of the areas of these rectangles. Gauss showed quite easily that the error made in this approximation is $P(x)=O(\sqrt{x})$.

In 1915, Hardy [49] proved that $P(x) \neq O\left(x^{1 / 4}\right)$, as $x$ tends to $\infty$. In other words, there is a sequence of points $\left\{x_{n}\right\}$ tending to $\infty$ on which $P\left(x_{n}\right) \neq O\left(x_{n}^{1 / 4}\right)$. (He actually proved a slightly stronger result.) In connection with his work on the circle problem, Hardy [49] proved that

$$
\sum_{0 \leq n \leq x}{ }^{\prime} r_{2}(n)=\pi x+\sum_{n=1}^{\infty} r_{2}(n)\left(\frac{x}{n}\right)^{1 / 2} J_{1}(2 \pi \sqrt{n x}),
$$

where $J_{1}(x)$ is the ordinary Bessel function of order 1. After Gauss, almost all efforts toward obtaining an upper bound for $P(x)$ have ultimately rested upon (13.2), and methods of estimating the approximating trigonometric series that is obtained from the asymptotic formula for $J_{1}(2 \pi \sqrt{n x})$ as $n \rightarrow \infty$. In 1906, W. Sierpinski [68] proved that $P(x)=O\left(x^{1 / 3}\right)$ as $x$ tends to $\infty$, and there have been many improvements in a century of work since then, with the best current result being $P(x)=O\left(x^{131 / 416+\epsilon}\right)$, for every $\epsilon>0$, due to M. N. Huxley [55] $\left(\frac{131}{416}=0.3149 \ldots\right)$. It is conjectured that $P(x)=O\left(x^{1 / 4+\epsilon}\right)$, for every $\epsilon>0$. In a footnote, Hardy remarks, "The form of this equation was suggested to me by Mr. S. Ramanujan, to whom I had communicated the analogous formula for $d(1)+d(2)+\cdots+d(n)$, where $d(n)$ is the number of divisors of $n . "$ In this same paper, Hardy relates a beautiful identity of Ramanujan connected with $r_{2}(n)$, namely, for $a, b>0,[49$, p. 283],

$$
\sum_{n=0}^{\infty} \frac{r_{2}(n)}{\sqrt{n+a}} e^{-2 \pi \sqrt{(n+a) b}}=\sum_{n=0}^{\infty} \frac{r_{2}(n)}{\sqrt{n+b}} e^{-2 \pi \sqrt{(n+b) a}},
$$

which is not given elsewhere in any of Ramanujan's published or unpublished work. These facts indicate that Ramanujan and Hardy undoubtedly had probing conversations about the circle problem.

On page 335 in [63], which is not in Ramanujan's lost notebook, but which is in one of those fragments published with the lost notebook, Ramanujan offers two identities 
involving Bessel functions. To state Ramanujan's claims, we need to first define

$$
F(x)= \begin{cases}{[x],} & \text { if } x \text { is not an integer, } \\ x-\frac{1}{2}, & \text { if } x \text { is an integer, }\end{cases}
$$

where, as customary, $[x]$ is the greatest integer less than or equal to $x$.

Entry 13.1 (p. 335). If $0<\theta<1$ and $x>0$, then

$$
\begin{aligned}
\sum_{n=1}^{\infty} F & \left(\frac{x}{n}\right) \sin (2 \pi n \theta)=\pi x\left(\frac{1}{2}-\theta\right)-\frac{1}{4} \cot (\pi \theta) \\
& +\frac{1}{2} \sqrt{x} \sum_{m=1}^{\infty} \sum_{n=0}^{\infty}\left\{\frac{J_{1}(4 \pi \sqrt{m(n+\theta) x})}{\sqrt{m(n+\theta)}}-\frac{J_{1}(4 \pi \sqrt{m(n+1-\theta) x})}{\sqrt{m(n+1-\theta)}}\right\} .
\end{aligned}
$$

Entry 13.1 was first proved by the author, S. Kim, and A. Zaharescu [29]. In [28], the same three authors proved (13.4) with the product $m n$ of the summation indices tending to $\infty$. Entry 13.1 was first established with the order of summation reversed from that prescribed by Ramanujan in (13.4) [34].

The Bessel functions in (13.4) bear a striking resemblance to those in (13.2), and so it is natural to ask if there is a connection between the two formulas. Berndt, Kim, and Zaharescu [28] proved the following corollary, as a consequence of their reinterpreted meaning of the double sum. It had been previously established (although not rigorously) by Berndt and Zaharescu in [34] as a corollary of their theorem on twisted divisor sums arising from Entry 13.1.

Corollary 13.2. For any $x>0$,

$$
\sum_{0 \leq n \leq x}{ }^{\prime} r_{2}(n)=\pi x+2 \sqrt{x} \sum_{n=0}^{\infty} \sum_{m=1}^{\infty}\left\{\frac{J_{1}\left(4 \pi \sqrt{m\left(n+\frac{1}{4}\right) x}\right)}{\sqrt{m\left(n+\frac{1}{4}\right)}}-\frac{J_{1}\left(4 \pi \sqrt{m\left(n+\frac{3}{4}\right) x}\right)}{\sqrt{m\left(n+\frac{3}{4}\right)}}\right\} .
$$

Can Corollary 13.2 be employed in place of (13.2) to effect an improvement in the error term for the circle problem? The advantage of (13.5) is that $r_{2}(n)$ does not appear on the right-hand side; the disadvantage is that one needs to estimate a double sum, instead of a single sum in (13.2).

The second identity on page 335 pertains to the famous Dirichlet divisor problem. Let $d(n)$ denote the number of positive divisors of the integer $n$. Define the "error term" $\Delta(x)$, for $x>0$, by

$$
\sum_{n \leq x}^{\prime} d(n)=x(\log x+(2 \gamma-1))+\frac{1}{4}+\Delta(x)
$$

where $\gamma$ denotes Euler's constant, and where the prime $/$ on the summation sign on the left side indicates that if $x$ is an integer, then only $\frac{1}{2} d(x)$ is counted. The famous Dirichlet divisor problem asks for the correct order of magnitude of $\Delta(x)$ as $x \rightarrow \infty$. Counting lattice points under a hyperbola, Dirichlet easily showed (13.6) with $\Delta(x)=$ 
$O(\sqrt{x})$. Estimates that have been established for the error term $\Delta(x)$ are similar to those that have been proved for $P(x)$. G. F. Voronoï [69] established a representation for $\Delta(x)$ in terms of Bessel functions with his famous formula

$$
\sum_{n \leq x}^{\prime} d(n)=x(\log x+(2 \gamma-1))+\frac{1}{4}+\sum_{n=1}^{\infty} d(n)\left(\frac{x}{n}\right)^{1 / 2} I_{1}(4 \pi \sqrt{n x}),
$$

where $x>0$ and

$$
I_{\nu}(z):=-Y_{\nu}(z)-\frac{2}{\pi} K_{\nu}(z)
$$

Here $Y_{1}(x)$ denotes the Bessel function of the second kind of order 1, and $K_{1}(x)$ denotes the modified Bessel function of order 1. Since its appearance in 1904, (13.7) has been the starting point for nearly all attempts at finding an upper bound for $\Delta(x)$.

We close this section by stating the second identity on page 335 of [63]. Note that the Bessel functions that appear below are the same as those in (13.7). Proofs of Entry 13.3 with the order of summation reversed from that given by Ramanujan in (13.9) and with the product of the summation indices $m$ and $n$ approaching $\infty$ can be found in the author's paper with Kim and Zaharescu [28]. See also [7, Chapter 2].

Entry 13.3 (p. 335). Let $F(x)$ be defined by (13.3), and let $I_{1}(x)$ be defined by (13.8). Then, for $x>0$ and $0<\theta<1$,

$$
\begin{aligned}
\sum_{n=1}^{\infty} F & \left(\frac{x}{n}\right) \cos (2 \pi n \theta)=\frac{1}{4}-x \log (2 \sin (\pi \theta)) \\
& +\frac{1}{2} \sqrt{x} \sum_{m=1}^{\infty} \sum_{n=0}^{\infty}\left\{\frac{I_{1}(4 \pi \sqrt{m(n+\theta) x})}{\sqrt{m(n+\theta)}}+\frac{I_{1}(4 \pi \sqrt{m(n+1-\theta) x})}{\sqrt{m(n+1-\theta)}}\right\} .
\end{aligned}
$$

\section{REFERENCES}

[1] G. E. Andrews, On the theorems of Watson and Dragonette for Ramanujan's mock theta functions, Amer. J. Math. 88 (1966), 454-490.

[2] G. E. Andrews, The fifth and seventh order mock theta functions, Trans. Amer. Math. Soc. 293 (1986), 113-134.

[3] G. E. Andrews, Ramanujan's fifth order mock theta functions as constant terms, in Ramanujan Revisited, G. E. Andrews, R. A. Askey, B. C. Berndt, K. G. Ramanathan, and R. A. Rankin, eds., Academic Press, Boston, 1988, pp. 47-56.

[4] G. E. Andrews and B. C. Berndt, Ramanujan's Lost Notebook, Part I, Springer, New York, 2005.

[5] G. E. Andrews and B. C. Berndt, Ramanujan's Lost Notebook, Part II, Springer, New York, 2009.

[6] G. E. Andrews and B. C. Berndt, Ramanujan's Lost Notebook, Part III, Springer, New York, 2012.

[7] G. E. Andrews and B. C. Berndt, Ramanujan's Lost Notebook, Part IV, Springer, New York, 2013.

[8] G. E. Andrews and F. G. Garvan, Dyson's crank of a partition, Bull. Amer. Math. Soc. 18 (1988), 167-171.

[9] G. E. Andrews and F. Garvan, Ramanujan's "lost" notebook. VI. The mock theta conjectures, Adv. Math. 73 (1989), 242-255.

[10] G. E. Andrews and D. Hickerson, Ramanujan's "Lost" Notebook VII: The sixth order mock theta functions, Adv. Math. 89 (1991), 60-105. 
[11] A. O. L. Atkin and H. P. F. Swinnerton-Dyer, Some properties of partitions, Proc. London Math. Soc. (3) 4 (1954), 84-106.

[12] B. C. Berndt, Modular transformations and generalizations of several formulae of Ramanujan, Rocky Mountain J. Math. 7 (1977), 147-189.

[13] B. C. Berndt, Analytic Eisenstein series, theta-functions, and series relations in the spirit of Ramanujan, J. Reine Angew. Math. 304 (1978), 332-365.

[14] B. C. Berndt, Ramanujan's Notebooks, Part I, Springer-Verlag, New York, 1985.

[15] B. C. Berndt, Ramanujan's Notebooks, Part II, Springer-Verlag, New York, 1989.

[16] B. C. Berndt, Ramanujan's Notebooks, Part III, Springer-Verlag, New York, 1991.

[17] B. C. Berndt, Ramanujan's Notebooks, Part IV, Springer-Verlag, New York, 1994.

[18] B. C. Berndt, Ramanujan's Notebooks, Part V, Springer-Verlag, New York, 1998.

[19] B. C. Berndt, What is a q-series?, in Ramanujan Rediscovered: Proceedings of a Conference on Elliptic Functions, Partitions, and q-Series in Memory of K. Venkatachaliengar: Bangalore, 1-5 June, 2009, Ramanujan Mathematical Society, Mysore, 2010, pp. 31-51.

[20] B. C. Berndt, The chief accountant and mathematical friend of Ramanujan - S. Narayana Iyer, Amer. Math. Monthly 118 (2011), 765-774.

[21] B. C. Berndt and P. R. Bialek, On the power series coefficients of certain quotients of Eisenstein series, Trans. Amer. Math. Soc. 357 (2005), 4379-4412.

[22] B. C. Berndt, P. R. Bialek, and A. J. Yee, Formulas of Ramanujan for the power series coefficients of certain quotients of Eisenstein series, International Mathematics Research Notices 2002, no. 21, 1077-1109.

[23] B. C. Berndt, H. H. Chan, S. H. Chan, and W.-C. Liaw, Ramanujan and cranks, in Theory and Applications of Special Functions. A Volume Dedicated to Mizan Rahman, M. E. H. Ismail and E. Koelink, eds., Springer, Dordrecht, 2005, pp. 77-98.

[24] B. C. Berndt, H. H. Chan, S. H. Chan, and W.-C. Liaw, Cranks - Really the final problem, Ramanujan J. 23 (2010), 3-15.

[25] B. C. Berndt, H. H. Chan, and L.-C. Zhang, Explicit evaluations of the Rogers-Ramanujan continued fraction, J. Reine Angew. Math. 480 (1996), 141-159.

[26] B. C. Berndt, G. Choi, Y.-S. Choi, H. Hahn, B. P. Yeap, A. J. Yee, H. Yesilyurt, and J. Yi Ramanujan's forty identities for the Rogers-Ramanujan functions, Memoir No. 880, American Mathematical Society 188 (2007).

[27] B. C. Berndt, B. Kim, and A. J. Yee, Ramanujan's lost notebook: Combinatorial proofs of identities associated with Heine's transformation or partial theta functions, J. Comb. Thy. Ser. A 117 (2010), 957-973.

[28] B. C. Berndt, S. Kim, and A. Zaharescu, Weighted divisor sums and Bessel function series, II, Adv. Math. 229 (2012), 2055-2097.

[29] B. C. Berndt, S. Kim, and A. Zaharescu, The circle problem and a double series of Bessel functions, Adv. Math. 236 (2013), 24-59.

[30] B. C. Berndt and K. Ono, Ramanujan's unpublished manuscript on the partition and tau functions with proofs and commentary, Sém. Lotharingien de Combinatoire 42 (1999), 63 pp.; in The Andrews Festschrift, D. Foata and G.-N. Han, eds., Springer-Verlag, Berlin, 2001, pp. 39-110.

[31] B. C. Berndt and R. A. Rankin, Ramanujan: Letters and Commentary, American Mathematical Society, Providence, RI, 1995; London Mathematical Society, London, 1995.

[32] B. C. Berndt and R. A. Rankin, Ramanujan: Essays and Surveys, American Mathematical Society, Providence, RI, 2001; London Mathematical Society, London, 2001.

[33] B. C. Berndt and A. J. Yee, Combinatorial proofs of identities in Ramanujan's lost notebook associated with the Rogers-Fine identity and false theta functions, Ann. of Combinatorics 7 (2003), 409-423.

[34] B. C. Berndt and A. Zaharescu, Weighted divisor sums and Bessel function series, Math. Ann. 335 (2006), 249-283.

[35] K. Bringmann and K. Ono, The $f(q)$ mock theta function conjecture and partition ranks, Invent. Math. 165 (2006), 243-266. 
[36] K. Bringmann and K. Ono, Dyson's ranks and Maass forms, Ann. Math. 171 (2010), 419-449.

[37] Y.-S. Choi, Tenth order mock theta functions in Ramanujan's lost notebook, Invent. Math. 136 (1999), 497-569.

[38] Y.-S. Choi, Tenth order mock theta functions in Ramanujan's lost notebook. II, Adv. Math. 156 (2000), 180-285.

[39] Y.-S. Choi, Tenth order mock theta functions in Ramanujan's lost notebook. III, Proc. London Math. Soc. (3) 94 (2007), 705-733.

[40] Y.-S. Choi, Tenth order mock theta functions in Ramanujan's lost notebook, IV, Trans. Amer. Math. Soc. 354 (2002), 147-159.

[41] F. J. Dyson, Some guesses in the theory of partitions, Eureka (Cambridge) 8 (1944), 10-15.

[42] F. J. Dyson, A walk through Ramanujan's garden, in Ramanujan Revisited, G. E. Andrews, R. A. Askey, B. C. Berndt, K. G. Ramanathan, and R. A. Rankin, eds., Academic Press, Boston, 1988, pp. 7-28; reprinted in [32, pp. 261-274].

[43] A. Folsom, A short proof of the mock theta conjectures using Maass forms, Proc. Amer. Math. Soc. 136 (2008), 4143-4149.

[44] A. Folsom, K. Ono, and R. C. Rhoades, Mock theta functions and quantum modular forms, Forum of Mathematics Pi 1 (2013), 1-27.

[45] A. Folsom, K. Ono, and R. C. Rhoades, Ramanujan's radial limits, Ramanujan 125, Contemporary Mathematics, vol. 627, K. Alladi, F. Garvan, and A. J. Yee, editors, American Mathematical Society, Providence, RI, 2014.

[46] F. G. Garvan, New combinatorial interpretations of Ramanujan's partition congruences mod 5, 7, and 11, Trans. Amer. Math. Soc. 305 (1988), 47-77.

[47] E. Grosswald, Die Werte der Riemannschen Zeta-funktion an ungeraden Argumentstellen, Nachr. Akad. Wiss. Göttingen (1970), 9-13.

[48] E. Grosswald, Comments on some formulae of Ramanujan, Acta Arith. 21 (1972), 25-34.

[49] G. H. Hardy, On the expression of a number as the sum of two squares, Quart. J. Math. (Oxford) 46 (1915), 263-283.

[50] G. H. Hardy and S. Ramanujan, Asymptotic formulae in combinatory analysis, Proc. London Math. Soc. (2) 17 (1918), 75-118.

[51] G. H. Hardy and S. Ramanujan, On the coefficients in the expansions of certain modular functions, Proc. Royal Soc. A 95 (1918), 144-155.

[52] D. Hickerson, A proof of the mock theta conjectures, Invent. Math. 94 (1988), 639-660.

[53] D. Hickerson, On the seventh order mock theta functions, Invent. Math. 94 (1988), 661-677.

[54] D. Hickerson and E. Mortenson, Hecke-type double sums, Appell-Lerch sums, and mock theta functions (I), Proc. London Math. Soc., to appear.

[55] M. N. Huxley, Exponential sums and lattice points. III, Proc. London Math. Soc. (3) 87 (2003), 591-609.

[56] K. Ono, Unearthing the visions of a master: Harmonic Maass forms and number theory, HarvardMIT Current Developments in Mathematics 2008, International Press, Somerville, MA, 2009, pp. $347-454$.

[57] H. Rademacher, On the partition function $p(n)$, Proc. London Math. Soc. 43 (1937), 78-84.

[58] S. Ramanujan, Highly composite numbers, Proc. London Math. Soc. 14 (1915), 347-400.

[59] S. Ramanujan, On certain arithmetical functions, Trans. Cambridge Philos. Soc. 22 (1916), 159184.

[60] S. Ramanujan, Some properties of $p(n)$, the number of partitions of $n$, Proc. Cambridge Philos. Soc. 19 (1919), 207-210.

[61] S. Ramanujan, Collected Papers, Cambridge University Press, Cambridge, 1927; reprinted by Chelsea, New York, 1962; reprinted by the American Mathematical Society, Providence, RI, 2000.

[62] S. Ramanujan, Notebooks (2 volumes), Tata Institute of Fundamental Research, Bombay, 1957.

[63] S. Ramanujan, The Lost Notebook and Other Unpublished Papers, Narosa, New Delhi, 1988.

[64] R. A. Rankin, George Neville Watson, J. London Math. Soc. 41 (1966), 551-565. 
[65] R. A. Rankin, Ramanujan's manuscripts and notebooks, Bull. London Math. Soc. 14 (1982), 81-97; reprinted in [32, pp. 117-128].

[66] R. A. Rankin, Ramanujan's manuscripts and notebooks II, Bull. London Math. Soc. 21 (1989), 351-365; reprinted in [32, pp. 129-142].

[67] L. J. Rogers, Second memoir on the expansion of certain infinite products, Proc. London Math. Soc. 25 (1894), 318-343.

[68] W. Sierpinski, O pewnem zagadnieniu z rachunku funkeyj asymptotycznych, Prace Mat. Fiz. 17 (1906), 77-118.

[69] G. F. Voronoï, Sur une fonction transcendante et ses applications à la sommation de quelques séries, Ann. École Norm. Sup. (3) 21 (1904), 207-267, 459-533.

[70] G. N. Watson, The final problem: an account of the mock theta functions, J. London Math. Soc. 11 (1936), 55-80; reprinted in [32, pp. 325-347].

[71] G. N. Watson, The mock theta functions (2), Proc. London Math. Soc. 42 (1937), 274-304.

[72] J. M. Whittaker, George Neville Watson, Biographical Memoirs of Fellows of the Royal Society 12 (1966), 521-530.

[73] S. Zwegers, Mock Theta Functions, Doctoral Dissertation, Universiteit Utrecht, 2002.

Department of Mathematics, University of Illinois, 1409 West Green Street, UrBANA, IL 61801, USA

E-mail address: berndt@illinois.edu 